\title{
PDII- Additional Discussion of the Dynamic Aperture
}

\author{
Norman Gelfand
}

July 15, 2002

\section{Introduction}

This note is in the nature of an addition to the dynamic aperture calculations found in the report on the Proton Driver, FERMILAB-TM-2169.

A extensive discussion of the Proton Driver lattice, as well as the nomenclature used to describe it can be found in TM-2169. Basically the proposed lattice is a racetrack design with the two arcs joined by two long straight sections. The straight sections are dispersion free.

Tracking studies were undertaken with the objective of computing the dynamic aperture for the lattice and some of the results have been incorporated into TM-2169. This note is a more extensive report of those calculations.

\section{Tune Dependence of the Dynamic Aperture}

With a low energy accelerator, such as the Proton Driver, space charge effects, as well as other effects arising from the charge of the bunches in the accelerator, result in a tune envelope, i.e. the different particles in the bunch have different betatron tunes. In the Proton Driver the tune spread can be as large as $\approx 0.2$. For this reason it is necessary for the dynamic aperture to be large not only at the nominal tune but over the expected region of the tune spread. We therefore need to determine the dynamic aperture as a function of the tune of the test particle. The method used to compute the dynamic aperture is described in TM-2169. In this section we calculate the dynamic aperture only for $\epsilon_{x}=\epsilon_{y}$.

Without doing a full calculation with space charge we cannot simulate the environment experienced by our test particle. We attempt to approximate 
that environment by adjusting the tune of the lattice.

The tunes are varied in the calculation by adjusting the strengths of the trim quadrupoles in the straight sections. Four families of trim quadrupoles and two sextupole families are used. The trim quadrupoles in the first four cells of the straights form two families, one with the $q d$ trim quads and the other with the $q f$ trim quads. The other two trim quadrupole families are formed from the trim quadrupoles in straight sections cells 5,6, and 7. With these families the tune can be adjusted over the range $0.9>\nu>0.5$.

The plot of dynamic aperture vs. tune (Figure 1) shows that the dynamic aperture is large $(>300 \pi m m m r)$ in the region of the plot with $\delta \nu \approx 0.2$ down and to the left of the nominal tunes of $\nu_{x}=0.747$ and $\nu_{y}=0.684$. Thus the dynamic aperture should be sufficient for particles within the expected tune envelope.

\section{Magnet Errors}

The magnets to be used in the new Proton Driver will, like all magnets, suffer from variations in their characteristics resulting from the production tolerances. It is difficult to know the size of these uncertainties without a detailed engineering study. In TM-2169 a criterion is established for magnet flatness. From these limits are derived the limits on the size of various multipoles. Despite the admonition that these limits are not to be treated as rms variations in multipole strengths, that is precisely what has been done here in order to study the effect of the multipoles on the dynamic aperture. These limits, interpreted as the $\sigma$ of the magnet errors, were used to generate distributions for the errors. The mean of the distributions, which would be the systematic errors of the magnets, were assumed to be zero. This method should over estimate the error fields and thus the dynamic aperture will be underestimated. Table I contains the estimates used for the $\sigma$ in the multipole moments of the magnetic field.

Table I

\begin{tabular}{|c|c|r|}
\hline Magnet type & Multipole & $\sigma$ \\
\hline Dipole & $b_{2}$ & $0.05 / m^{2}$ \\
\hline Dipole & $b_{4}$ & $802 / m^{4}$ \\
\hline Quadrupole & $b_{3}$ & $0.465 / m^{3}$ \\
\hline Quadrupole & $b_{4}$ & $6.10 / m^{4}$ \\
\hline
\end{tabular}


$(\varepsilon / \pi)_{n x} \vee S \nu_{x}$ and $\nu_{y}$

Input file-gbpd_n2.tevbin

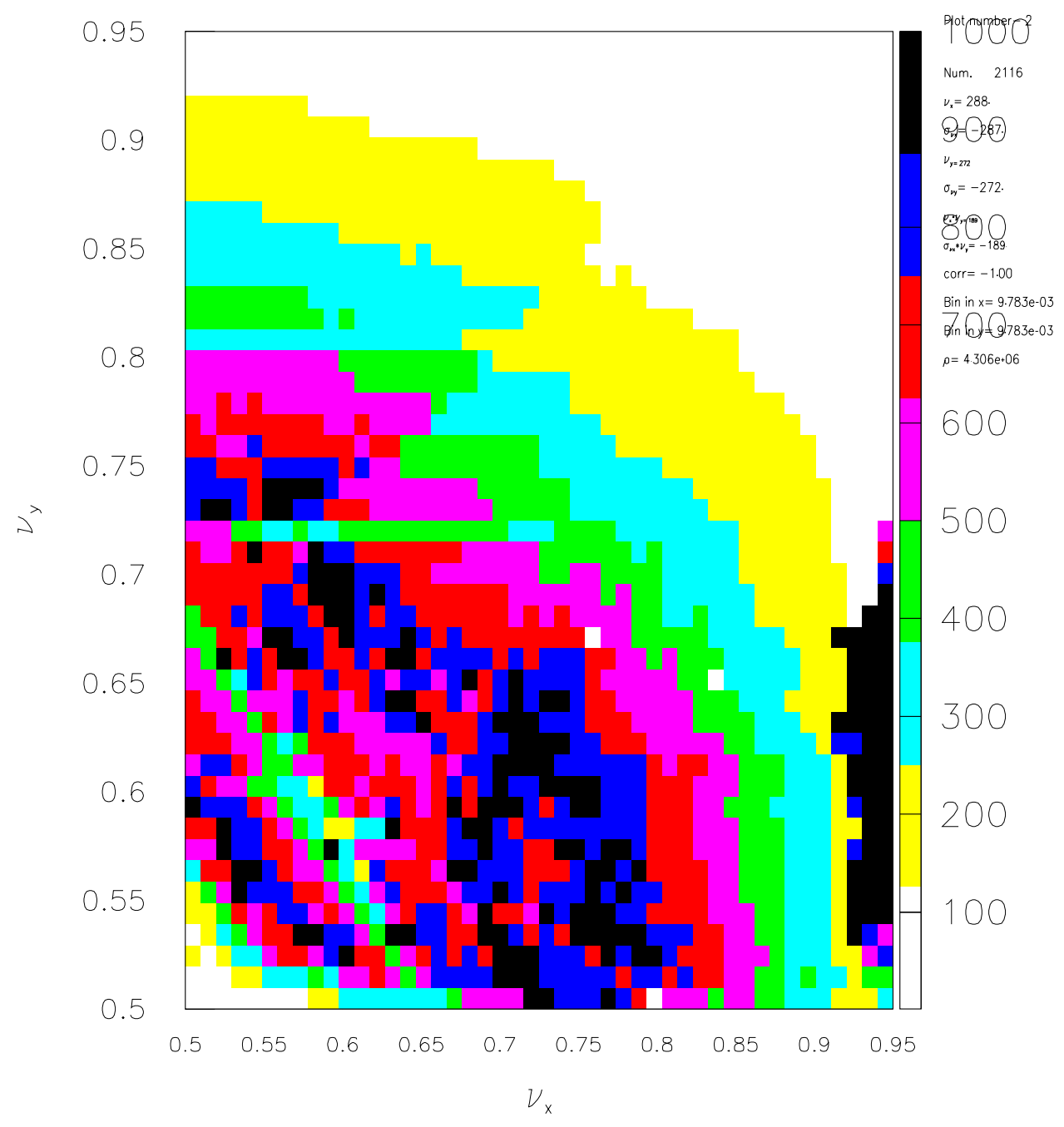

Figure 1: Dynamic Aperture as a Function of Tune. 
Thirty different sets of multipole coefficients were constructed. The dynamic aperture was calculated for each set of multipoles. The values of the multipoles assigned to the individual magnets in the lattice were generated using a random number generator which generated a Gaussian distribution, with the appropriate $\sigma$, and a mean of zero. The results for the calculated dynamic aperture are shown in Figure 2. It is clear that the multipoles significantly reduce the dynamic aperture but it remains $>300 \pi m m m r$. The calculated dynamic aperture satisfies the requirement that the dynamic aperture be at least 3 times the beam emittance $(>3 \epsilon(=40 \pi m m m r))$.

\section{Conclusion}

The dynamic aperture of the proposed lattice for the Proton Driver should be large enough $(>3 \cdot \epsilon)$ over the tune footprint expected from the space charge effects. The inclusion of magnet errors reduces the dynamic aperture but it still exceeds the criterion of being $>3 \cdot \epsilon$. 


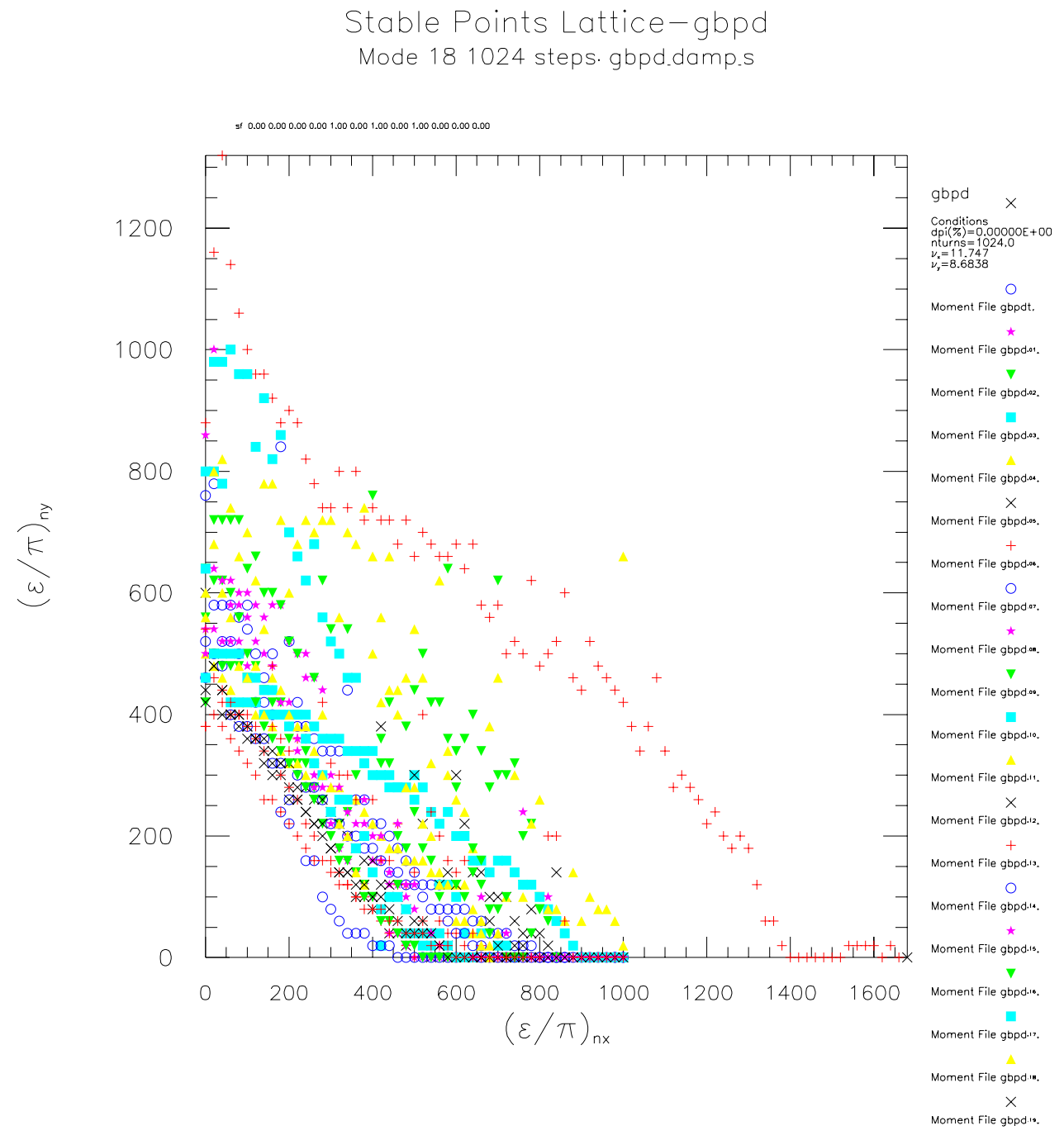

Figure 2: Dynamic Aperture for Different Magnet Errơrsers. file gopde. 